\title{
USO DE CATETERES VESICAIS PARA CATETERISMO INTERMITENTE LIMPO: SATISFAÇÃO DA PESSOA COM LESÃO MEDULAR*
}

Gisela Maria Assis ${ }^{1}$, Aline Fernanda Negri ${ }^{2}$, Sabrina Alves Veiga Tung ${ }^{3}$, Camila Francine Galli Saccomani ${ }^{4}$, Auristela Duarte de Lima Moser ${ }^{5}$, Gisele Regina de Azevedo ${ }^{6}$, Gislaine de Melo Meira ${ }^{7}$

${ }^{1}$ Enfermeira. Mestre em Tecnologia em Saúde. Hospital de Clínicas da Universidade Federal do Paraná. Curitiba, PR, Brasil. ${ }^{2}$ Enfermeira. Mestre em Educação das Profissões de Saúde. UBS Sorocaba. Sorocaba, SP, Brasil.

${ }^{3}$ Enfermeira. Estomaterapeuta. Hospital São Camilo. Sorocaba, SP, Brasil.

${ }^{4}$ Enfermeira. Estomaterapeuta. Intermedica - Hospital Modelo. Sorocaba, SP, Brasil.

${ }^{5}$ Fisioterapeuta. Doutora em Etgonomia. Pontifícia Universidade Católica do Paraná. Curitiba, PR, Brasil.

${ }^{6}$ Enfermeira. Doutora em Enfermagem na Saúde do Adulto. Pontifícia Universidade Católica de São Paulo. Sorocaba, SP, Brasil.

${ }^{7}$ Enfermeira. Estomaterapeuta. Maximed sul. Florianópolis, SC, Brasil.

RESUMO: Objetivou-se com este estudo avaliar a satisfação de pessoas com lesão medular com a utilização de cateter convencional, hidrofílico e pré-lubrificado com bolsa na realização de cateterismo intermitente limpo. Pesquisa exploratório-descritiva, de corte transversal com 59 pessoas que tiveram lesão medular, atendidas em centros de reabilitação de três estados brasileiros no período de janeiro a abril de 2012. Foi utilizado o questionário de avaliação de percepção de atributos do cateter e o questionário adaptado de satisfação em experiência de consumo. O cateter convencional não foi estatisticamente superior em nenhum aspecto avaliado. O cateter hidrofílico foi considerado superior ao convencional para abertura da embalagem e deslizamento na inserção. O cateter pré-lubrificado com bolsa foi superior ao convencional em abertura da embalagem, introdução, deslizamento e retirada. A indicação do cateter ideal depende da avaliação individualizada com lesão medular, suas dificuldades, potencialidades e preferências.

DESCRITORES: Cateterismo uretral intermitente; Paraplegia; Satisfação do paciente.

\section{USE OF URINARY CATHETERS FOR CLEAN INTERMITTENT CATHETERIZATION: SATISFACTION OF PATIENTS WITH BONE MARROW INJURY}

\begin{abstract}
The objective in this study was to assess the use of the conventional catheter, hydrophilic catheter and pre-lubricated catheter with bag for clean intermittent catheterization. Exploratory-descriptive cross-sectional research involving 59 bone marrow injury patients attended at rehabilitation centers of three Brazilian states between January and April 2012. The questionnaire to assess the perceived catheter attributes and the adapted consumption experience satisfaction questionnaire were used. The conventional catheter was not statistically superior in any aspect assessed. The hydrophilic catheter was considered superior to the conventional for package opening and sliding during insertion. The pre-lubricated catheter with bag was superior to the conventional for package opening, introduction, sliding and removal. The indication of the ideal catheter depends on the individual assessment of the patients, their difficulties, potentials and preferences.
\end{abstract}

DESCRIPTORS: Intermittent urethral catheterization; Paraplegia; Patient satisfaction.

\section{USO DE CATÉTERES VESICALES PARA CATETERISMO INTERMITENTE LIMPIO: SATISFACCIÓN DE LA PERSONA CON LESIÓN MEDULAR}

RESUMEN: Estudio cuya finalidad fue evaluar la satisfacción de personas con lesión medular con la utilización de catéter convencional, hidrofílico y prelubrificado con bolsa en la realización de cateterismo intermitente limpio. Investigación exploratoria descriptiva, transversal con 59 personas que tuvieron lesión medular, atendidas en centros de rehabilitación de tres estados brasileños en el periodo de enero a abril de 2012. Fue utilizado el cuestionario de evaluación de percepción de atributos de catéter y el cuestionario adaptado de satisfacción en experiencia de consumo. El catéter convencional no fue estadisticamente superior en ningun aspecto evaluado. El catéter hidrofílico fue considerado superior al convencional para abertura del embalaje y deslizamiento en la inserción. El catéter prelubrificado con bolsa fue superior al convencional en abertura del embalaje, introducción, deslizamiento y retirada. La indicación del catéter ideal depende de la evaluación individualizada con lesión medular, sus dificultades, potencialidades y preferencias.

DESCRIPTORES: Cateterismo uretral intermitente; Paraplegia; Satisfacción del paciente. *Artigo extraído da e dissertação: “Satisfação de pessoas com lesão medular com a utilização de cateteres vesicais de diferentes
tecnologias." Pontifícia Universidade Católica do Paraná. 2012.

Autor Correspondente:

Gisela Maria Assis

Hospital de Clínicas da UFPR

R. General Carneiro 181 - 80.060-900 - Curitiba, PR, Brasil

E-mail: giassis83@gmail.com
Recebido: 29/05/2015

Finalizado: 11/09/2015 


\section{INTRODUÇÃO}

Uma das consequências da lesão medular é a disfunção neurológica do trato urinário inferior (DNTUI). Esta resulta em esvaziamento incompleto da bexiga e tem como complicações as infecções do trato urinário (ITU), refluxo vesico ureteral, hidronefrose e perda da função renal. O cateterismo intermitente é o tratamento de escolha para prevenção das complicações da DNTUI, e trata-se do esvaziamento periódico da bexiga pela introdução de um cateter pela uretra ou canal cateterizável ${ }^{(1)}$.

O cateterismo intermitente limpo (CIL), proposto por Lapides em 1970, consiste na utilização de técnica e material não-estéreis, apenas com limpeza das mãos e região genital ${ }^{(2)}$. Observou-se que as ITU eram causadas por distensão vesical e estase urinária e não pela assepsia da técnica ${ }^{(3)}$.

Um número crescente de publicações comparando diferentes cateteres vesicais para CIL demonstra a importância do tema, o que é corroborado também por autores que afirmam que a indicação do cateter mais adequado à necessidade e preferências do cliente é um fator determinante para sua adesão à técnica ${ }^{(4)}$.

Estudos comparativos utilizando diferentes cateteres não encontraram diferença entre os convencionais e pré-lubrificados, no que diz respeito ao sangramento e ITU ${ }^{(5-6)}$, porém foram citadas diferenças para microtrauma uretral, leucocitúria e, de maneira mais expressiva, para satisfação do usuário(7).

A limitação de acesso a diferentes cateteres no cenário nacional suscita a necessidade de elaboração de políticas públicas que favoreçam o direito de escolha dos usuários. Autores citam que o conhecimento do grau de atendimento às expectativas do usuário tem como benefício a produção de subsídios para elaboração destas políticas.

Assim, objetivou-se avaliar a satisfação da pessoa com lesão medular na utilização de três cateteres vesicais, de diferentes tecnologias, comparando e analisando diferentes atributos destes, na utilização de cateteres convencionais, hidrofílicos e pré-lubrificados.

\section{MÉTODO}

Estudo exploratório, descritivo, de corte transversal, com abordagem quantitativa. O estudo foi realizado em três centros de reabilitação de três grandes cidades dos estados de São Paulo, Paraná e Santa Catarina. O período de coleta de dados foi de janeiro a abril de 2012.

A amostra, por conveniência, foi composta por 59 indivíduos, tendo como critérios de inclusão: ser maior de 18 anos, possuir lesão medular, CIL como forma de esvaziamento vesical há pelo menos três meses, ser alfabetizado, vinculado com os locais de coleta e sem dificuldade de se autocateterizar.

Para a coleta de dados foram utilizados três instrumentos. O primeiro para caracterização da amostra, preenchido pelo pesquisador no primeiro contato com o participante. Os outros dois instrumentos (como questionário) eram preenchidos pelo participante ao final do período de avaliação dos cateteres.

O instrumento de percepção do cliente com o cateter vesical utilizado foi desenvolvido por enfermeiras estomaterapeutas. Possuía itens específicos relacionados aos cateteres, como: abertura da embalagem, manipulação, deslizamento na uretra, retirada, tempo de procedimento, entre outros. As questões eram respondidas em uma escala Likert de cinco pontos, e as respostas eram de "muito ruim" a "muito bom" ou "muito difícil" a "muito fácil". Considerou-se como satisfeito o sujeito que assinalou as respostas: "bom" e "muito bom", "fácil" e "muito fácil", tendo em vista que o "razoável", ou nota três, é a linha de corte entre satisfação e insatisfação.

O outro instrumento de avaliação foi uma adaptação do instrumento de Encantamento do Cliente $^{(8)}$, que contava com itens gerais, relacionados à experiência de consumo de um produto como: resposta às expectativas e desejos, percepção de qualidade e performance do produto e tendência a comentários positivos sobre ele. As respostas eram assinaladas em uma escala de um a sete, que ia desde "discordo totalmente" até "concordo totalmente". Considerando encantamento o nível mais alto de satisfação, ou, nota sete de concordância, considerou-se como satisfeito o indivíduo que assinalou as notas maior ou igual a cinco, no item avaliado.

Cada sujeito avaliou três cateteres vesicais. O cateter convencional, de Cloreto de Polivinila (PVC) sem lubrificação, já utilizado por todos eles há mais de três meses, foi avaliado retrospectivamente. O cateter hidrofílico e o pré- 
lubrificado com bolsa foram fornecidos a cada um em número suficiente para 24 horas de uso, de acordo com o intervalo observado entre as cateterizações.

Os novos cateteres avaliados foram: cateter de poliuretano, com lubrificação hidrofílica de segunda geração, de polivinilpirrolidona (PVP) e solução salina isotônica (SSI), e cateter de PVC, lubrificado por solução de glicerina e água, revestido por um filme de polietileno flexível, que permite técnica sem toque, acoplado a uma bolsa coletora de urina descartável e drenável, com capacidade de $2000 \mathrm{ml}$.

Os indivíduos recebiam orientações sobre a utilização de cada cateter e preenchimento de cada instrumento, levavam para casa os dois instrumentos de avaliação para cada cateter que seria avaliado, bem como cateteres hidrofílicos e pré-lubrificados com bolsa suficientes para utilizarem cada tipo por 24 horas. Depois da utilização e preenchimento, os indivíduos retornavam ao local de estudo para devolução dos instrumentos preenchidos.

A análise estatística foi realizada por meio do programa Statística versão 8.0. A comparação dos cateteres em pares foi realizada utilizandose o teste binomial e a comparação de grupos, definidos por sexo feminino e masculino e por nível de lesão, e foi realizada por meio da aplicação do teste não-paramétrico de Mann-Whitney. Para variáveis qualitativas considerou-se o teste de Qui-quadrado ou o teste exato de Fisher. Valores de $p<0,05$ indicaram significância estatística.

O projeto de pesquisa foi aprovado pelo CEPPUC/PR sob parecer n. ${ }^{\circ}$ 5359/11. Os sujeitos foram devidamente informados ética e tecnicamente sobre todos os aspectos do estudo.

\section{RESULTADOS}

Referente à caracterização da amostra estudada, a idade dos sujeitos variou de 18 a 65 anos, com uma média de 34,6 anos $( \pm 11,8)$. O sexo masculino foi predominante, com $76,3 \%(n=45)$. A escolaridade se mostrou distribuída em vários níveis. Quanto ao nível de escolaridade, o ensino médio completo foi predominante com 37,3\% $(\mathrm{n}=22)$, seguido por fundamental incompleto com 30,5\% (n=18). Quanto ao nível de lesão, 67,8\% $(n=40)$ eram paraplégicos. A causa mais frequente de lesão foi ferimento por arma de fogo, seguido por acidente automobilístico e queda de nível. O tempo decorrido desde a lesão medular variou entre 0,6 a 28 anos, com média de 7,1 anos $( \pm 6,2)$.
Quanto à sensibilidade uretral, autorreferida, $44,1 \%(n=26)$ mantinham-na preservada.

Para o item abertura da embalagem, o percentual de usuários satisfeitos com o cateter convencional foi de $54,2 \%$, para o cateter hidrofílico foi de $74,6 \%$ e para o conjunto para CIL de $71,2 \%$. Vale citar que o cateter hidrofílico apresentou o menor percentual de usuários insatisfeitos $(1,7 \%)$. Considerando as médias de cada cateter, houve diferença estatisticamente significativa entre os cateteres hidrofílico e prélubrificado com bolsa e o convencional, conforme apresentado no Quadro 1.

Na avaliação de manipulação do cateter, não houve diferença estatisticamente significativa na comparação dois a dois. O percentual de satisfação para este item foi de $59,3 \%$ para o convencional, $61 \%$ para o hidrofílico e $67,8 \%$ para o pré-lubrificado com bolsa.

Para introdução do cateter na uretra, houve diferença estatisticamente significativa somente para a comparação entre o cateter convencional e o pré-lubrificado com bolsa, sendo a média do segundo superior, conforme demonstrado no Quadro 01. O percentual de satisfação do prélubrificado com bolsa foi de $72,9 \%$, do cateter hidrofílico $61 \%$ e do cateter convencional $45,8 \%$. O cateter hidrofílico teve o maior percentual de indivíduos insatisfeitos neste item (23,7\%).

O cateter hidrofílico obteve o maior percentual de satisfação com o deslizamento do cateter na uretra, com $89,8 \%$ dos participantes e um percentual de insatisfação de $3,4 \%$. O percentual de usuários satisfeitos com o deslizamento do pré-lubrificado com bolsa foi de $83 \%$ e do cateter convencional 37,3\%, com 33,9\% de usuários insatisfeitos. Comparando as médias dos cateteres, houve diferença estatisticamente significativa $(p<0,001)$ para os cateteres hidrofílico e pré-lubrificado em relação ao convencional (Quadro 1).

$\mathrm{Na}$ avaliação de retirada do cateter, $64,4 \%$ referiram estar satisfeitos com $\mathrm{O}$ cateter convencional; $81,4 \%$ com o cateter hidrofílico e $88,1 \%$ com o pré-lubrificado com bolsa. A comparação entre as médias resultou em diferença estatisticamente significativa entre o cateter convencional e o pré-lubrificado com bolsa, como apresentado no Quadro 1.

Quanto à sensação de segurança na utilização do cateter, a satisfação referida foi de $64,4 \%$ com o cateter convencional; $72,1 \%$ com o hidrofílico e $88,1 \%$ satisfeitos com o pré-lubrificado com 
Quadro 1 - Média de satisfação de pessoas com lesão medular com o uso de cateteres vesicais de diferentes tecnologias - Sorocaba (SP), Curitiba (PR), Joinville (SC), Brasil, 2012

\begin{tabular}{|lcccccc|}
\hline $\begin{array}{l}\text { Atributo avaliado } \\
\text { (valores para a média) }\end{array}$ & $\mathbf{C 1}$ & $\mathbf{C 2}$ & $\mathbf{C 3}$ & $\begin{array}{c}\text { Valor de } \\
\text { p* para a alor de } \\
\text { diferença } \\
\text { C1xC2 }\end{array}$ & $\begin{array}{c}\text { Valor de } \\
\text { p* para a } \\
\text { diferença } \\
\text { C1xC3 }\end{array}$ & $\begin{array}{c}\text { p* para a } \\
\text { diferença } \\
\text { C2xC3 }\end{array}$ \\
\hline Escala de avaliação da percepção do cliente quanto ao cateter no CIL & & & & & \\
\hline Abertura (01 a 05) & 3,5 & 4,1 & 3,9 & 0,003 & 0,046 & 0,309 \\
\hline Manipulação (01 a 05) & 3,6 & 3,6 & 3,9 & 0,76 & 0,085 & 0,124 \\
\hline Introdução (01 a 05) & 3,3 & 3,6 & 3,9 & 0,157 & 0,004 & 0,114 \\
\hline Deslizamento (01 a 05) & 3 & 4,3 & 4,2 & $<0,001$ & $<0,001$ & 0,612 \\
\hline Retirada (01 a 05) & 3,7 & 4 & 4,2 & 0,103 & 0,001 & 0,15 \\
\hline Segurança (01 a 04) & 2,8 & 2,8 & 3,3 & 0,809 & $<0,001$ & 0,001 \\
\hline Nota/conceito (01 a 05) & 3,4 & 3,8 & 4,3 & 0,063 & $<0,001$ & 0,007 \\
\hline Escore (07 a 34) & 23,3 & 26,2 & 27,9 & 0,004 & $<0,001$ & 0,066 \\
\hline Adaptação da escala de avaliação de encantamento/satisfação - Adaptada de Almeida e Nique $(2007)$ & \\
\hline Expectativas (01 a 07) & 4,1 & 4,8 & 5,5 & 0,072 & $<0,001$ & 0,039 \\
\hline Satisfação (01 a 07) & 3,9 & 5 & 5,6 & 0,001 & $<0,001$ & 0,046 \\
\hline Qualidade (01 a 07) & 4 & 5,4 & 5,8 & $<0,001$ & $<0,001$ & 0,176 \\
\hline Performance (01 a 07) & 3,9 & 5,1 & 5,5 & 0,001 & $<0,001$ & 0,109 \\
\hline Desejos(01 a 07) & 4,2 & 4,9 & 5,4 & 0,05 & 0,001 & 0,138 \\
\hline Comentários positivos (01 a 07) & 4,4 & 4,9 & 5,5 & 0,184 & 0,001 & 0,093 \\
\hline Recomendação a amigo (01 a 07) & 4,5 & 4,9 & 5,5 & 0,302 & 0,004 & 0,041 \\
\hline
\end{tabular}

*Teste não-paramétrico de Wilcoxon, $\mathrm{p}<0,05$.

C1: cateter convencional de PVC sem lubrificação prévia.

C2: cateter de poliuretano com lubrificação hidrofílica.

C3: cateter pré lubrificado com bolsa.

bolsa. Na comparação de médias, apresentada no Quadro 1, o conjunto para CIL foi estatisticamente superior ao cateter convencional e ao cateter hidrofílico.

Para o item desconforto durante a introdução do cateter, $56 \%$ o referiram com o cateter convencional, $32 \%$ com o cateter hidrofílico e $20 \%$ com o pré-lubrificado com bolsa, resultando em diferença estatisticamente significativa entre o cateter convencional e o conjunto para CIL $(p=0,022)$. Vale ressaltar que somente os indivíduos com sensibilidade uretral preservada responderam a essa questão.

$\mathrm{Na}$ nota/conceito atribuída a cada cateter; $50,8 \%$ avaliou o cateter convencional como "bom" ou "muito bom". Esse percentual foi de $69,5 \%$ para o cateter hidrofílico e $78 \%$ para o prélubrificado com bolsa, sendo que este obteve um percentual de apenas $1,7 \%$ que o classificou como "ruim" ou "muito ruim". A média obtida foi estatisticamente superior para o pré-lubrificado com bolsa em relação ao cateter convencional e ao cateter hidrofílico.

O escore dos cateteres, obtido a partir da soma dos itens pontuáveis do instrumento um, resultou em uma média de 23,3 para o cateter convencional; 26,2 para o cateter hidrofílico e 27,9 para o prélubrificado com bolsa. Desta forma, nenhum dos cateteres avaliados foi classificado, de acordo com o instrumento, como "muito bom" (escores de 28 a 34), todos ficaram classificados como "bom" (21 a 27 pontos). Os escores apresentados resultaram em superioridade significativa para os cateteres pré-lubrificados (hidrofílico e pré-lubrificado com bolsa) em relação ao cateter convencional.

A respeito do tempogasto para o procedimento, não houve diferença estatisticamente significativa na comparação entre os cateteres. A média de tempo dispendido para o cateter convencional foi de 5,6 minutos, para o cateter hidrofílico de 4,9 e para o pré-lubrificado com bolsa de 4,5 minutos.

Os dados obtidos a partir da análise do instrumento de Encantamento do cliente apresentaram comportamento semelhante, sendo um percentual menor de pessoas satisfeitas com o cateter convencional, maior para o cateter hidrofílico e ainda maior para o cateter prélubrificado com bolsa. As médias de cada cateter 
e a comparação das mesmas com valor de p estão apresentadas no Quadro 1.

$\mathrm{Na}$ avaliação de resposta às expectativas pré-consumo, o cateter pré-lubrificado com bolsa obteve maior média e percentual de satisfação entre os participantes, resultando em superioridade significativa em relação aos outros cateteres. O mesmo aconteceu na avaliação de satisfação pós-consumo. Porém neste item, o cateter hidrofílico também foi estatisticamente superior ao cateter convencional. $\mathrm{Q} u$ a $\mathrm{n} \mathrm{t} \mathrm{o}$ à qualidade e desempenho dos cateteres e resposta aos desejos do usuário, o cateter hidrofílico e pré-lubrificado com bolsa foram superiores ao convencional. Não houve diferença estatisticamente significativa na comparação entre os cateteres pré-lubrificados.

No item referente à propensão do indivíduo a fazer comentários positivos, houve significância estatística somente para o cateter pré-lubrificado com bolsa em relação ao cateter convencional. Esta mesma diferença foi observada para a propensão à recomendação do produto a um amigo, sendo que neste item, a superioridade do cateter pré-lubrificado com bolsa se apresentou também na comparação com o cateter hidrofílico.

Para todas as questões foi realizada a comparação de respostas entre homens e mulheres e entre pessoas com lesões até T4 e a partir de T5. Nenhum destes cruzamentos de variáveis resultou em diferença considerável. Respondendo às questões norteadoras do estudo, pode-se afirmar que há diferença na satisfação de pessoas com lesão medular quanto à utilização de cateteres vesicais convencionais e pré-lubrificados. Existe também diferença na satisfação desta população quanto ao uso de diferentes cateteres pré-lubrificados. Porém, a preferência do usuário não é por um tipo de cateter e sim por características específicas encontradas em cada um.

O cateter convencional não foi estatisticamente superior em nenhum item avaliado. $O$ cateter hidrofílico foi superior ao convencional com significância para abertura da embalagem, deslizamento na uretra, escore geral, satisfação, qualidade e performance. $\mathrm{O}$ cateter prélubrificado com bolsa foi superior ao convencional nos itens: abertura, introdução, deslizamento, retirada, escore geral, expectativas, satisfação, qualidade, performance, resposta aos desejos pré consumo e propensão a comentários positivos e recomendação do produto. Nos itens segurança, expectativa, satisfação e propensão a recomendar o produto, foram superiores também ao cateter hidrofílico.

Apesar dos instrumentos utilizados não avaliarem, de forma direta, os aspectos dos cateteres que podem influenciar no grau de satisfação, pode-se observar indiretamente essas variáveis, por meio da avaliação do diferencial apresentadopelocateterqueobtevesuperioridade de avaliação em cada item. Assim, pode-se sugerir que estes aspectos são: artifícios que facilitem a manipulação do cateter, desde a abertura da embalagem até a retirada, reservatório coletor de urina acoplado, lubrificação homogênea que proporcione deslizamento suave e não interfira na firmeza de introdução do cateter.

\section{DISCUSSÃO}

O cateter hidrofílico, que obteve o melhor resultado para abertura da embalagem, possui como diferenciais para este item, um adesivo na parte posterior da embalagem, que permite colálo em superfície lisa e abri-lo ocupando apenas uma das mãos, e um anel de abertura como auxílio para pessoas com menor destreza manual. O segundo cateter melhor avaliado foi o prélubrificado com bolsa, que possui uma face de plástico e outra de papel, em um ponto central do local para abertura, a face de papel é mais curta, facilitando a separação entre as duas.

Autores não encontraram diferença para abertura da embalagem do cateter convencional e hidrofílico, sendo que o estudo contava com uma versão do cateter hidrofílico para mulheres, com abertura diferenciada, o que pode ter contribuído para a diferença encontrada ${ }^{(9)}$.

Quanto à manipulação do cateter, não houve diferença estatisticamente significativa entre a avaliação dos indivíduos para os três cateteres, sendo que alguns indivíduos citaram dificuldade de manipulação do hidrofílico por não poder segurar em qualquer local da extensão do cateter. Outros consideraram o cateter pré-lubrificado com bolsa muito longo.

Outros estudos também não encontraram diferença na manipulação de cateteres prélubrificado e hidrofílico ${ }^{(10)}$, ou convencional e hidrofílico ${ }^{(6)}$. Em estudo que comparou diferentes marcas de cateteres hidrofílicos ${ }^{(11)}$, dois participantes de 20, consideraram escorregadio o hidrofílico usado no presente estudo e um o considerou pegajoso.

Autores relataram que os cateteres hidrofílicos 
podem ser muito escorregadios, dificultando a manipulação se não tiver uma proteção em que possa ser manuseado. Os mesmos autores relatam que os cateteres com bolsa acoplada podem ser difíceis de manipular por pessoas com limitação de destreza manual ${ }^{(12)}$.

A ausência de diferença significativa para manipulação entre os três cateteres pode estar associada à familiaridade que os indivíduos possuem com o cateter convencional, fato que facilita a manipulação mesmo sem artifícios facilitadores.

A superioridade na avaliação de introdução na uretra para o pré-lubrificado com bolsa pode ter sido motivada pela possibilidade de realização de técnica sem toque. O filme de polietileno de baixa densidade que recobre diretamente o cateter não é retirado no momento da técnica, servindo de revestimento protetor por meio do qual o sujeito segura o cateter. Por outro lado, o percentual de insatisfação para o cateter hidrofílico pode estar relacionado à impossibilidade de segurar o cateter em qualquer local de seu comprimento, não permitindo firmeza em sua introdução.

Estudo demonstrou dificuldade de crianças introduzirem o cateter hidrofílico, comparado ao convencional. A maior dificuldade foi pelo fato de ele ser muito escorregadio ${ }^{(9)}$, e esta pode ter sido a mesma que motivou sua pior avaliação em relação ao pré-lubrificado com bolsa, considerando que cinco sujeitos o classificaram como "ruim" ou "muito ruim" por ele ser muito liso.

Em estudo em que foram comparados apenas cateteres hidrofílicos ${ }^{(11)}, 85 \%$ dos usuários consideraram boa a introdução do hidrofílico e $90 \%$ considerou boa a introdução da outra marca comparada, com algumas referências de estes serem muito escorregadios ou pegajosos. Autores observaram diferença estatisticamente significativa para conforto e facilidade na introdução do cateter, com superioridade para o cateter hidrofílico em relação ao convencional ${ }^{(13)}$. Outros, tendocomoamostracrianças estomizadas, não observaram tal diferença ${ }^{(6)}$. Comparando cateteres pré-lubrificados hidrofílicos e não hidrofílicos com bolsa de urina acoplada, autores observaram melhor avaliação para o hidrofílico ${ }^{(10)}$.

O cateter hidrofílico apresentou o maior percentual de satisfação para deslizamento na uretra, possivelmente pela sua tecnologia de lubrificação. $O$ cateter é revestido por polivinilpirrolidona (PVP), um polímero com capacidade de absorver até 10 vezes o seu próprio peso. Vem imerso em Solução Salina Isotônica (SSI) que aumenta a osmolaridade, resultando em um revestimento isotônico.

Estudos randomizados e controlados demonstram resultados favoráveis dos cateteres hidrofílicos na prevenção da hematúria e bacteriúria ${ }^{(1)}$, indicando sua capacidade de deslizamento atraumático através da uretra, capaz de evitar microtraumas na progressão.

O cateter pré-lubrificado com bolsa de lubrificação não hidrofílica teve superioridade estatística em relação ao cateter convencional. Isso demonstra que não somente lubrificação de segunda geração, mas uma lubrificação prévia e homogênea contribuem para um bom deslizamento. O cateter convencional obteve um percentual baixo de satisfação, considerando que os sujeitos lubrificam o cateter com lidocaína. Observou-se que a lubrificação manual não é suficiente para gerar um deslizamento suave.

O alto percentual de satisfação durante a retirada do cateter pré-lubrificado com bolsa pode ter sido motivado pela presença de bolsa coletora de urina com válvula antirrefluxo, o que evita respingos. Por outro lado, o cateter hidrofílico obteve o maior percentual de sujeitos insatisfeitos, justificado pelos participantes pelo fato do cateter não poder ser dobrado para retirada. Assim, quando não se conecta a bolsa coletora, há um risco de derramamento da urina que ficou na luz do cateter.

Autores que compararam cateteres hidrofílicos e pré-lubrificados, ambos com bolsa, obtiveram a melhor avaliação para o hidrofílico ${ }^{(10)}$. Em outro estudo comparativo com diferentes cateteres hidrofílicos, $84 \%$ dos participantes consideraram boa sua retirada e $5 \%$ da amostra o considerou muito pegajoso para a retirada. Na retirada pós CIL em estomas urinários, não houve diferença entre cateter convencional e hidrofílico ${ }^{(6)}$.

Acredita-se que a soma de alguns atributos influenciou positivamente a avaliação, como: a possibilidade de realizar a técnica sem tocar no cateter, gerando um sentimento de segurança quanto ao risco de infecção, a possibilidade de segurar próximo ao orifício de drenagem de urina e a facilidade de ter a bolsa de coleta de urina acoplada ao sistema, evitando desconexões ou respingos.

Reforçando a facilidade de ter uma bolsa conectada ao cateter para drenagem da urina, autores descreveram as barreiras encontradas para implementação do CIL para pessoas com 
lesão medular ${ }^{(4)}$. Entre elas, encontra-se a dificuldade de acesso e adequação de banheiros públicos, problema solucionado por alguns dos sujeitos estudados pelo uso de cateteres com bolsas coletoras acopladas.

O cateter hidrofílico, apesar de ter obtido o melhor resultado para deslizamento, foi superado no item desconforto pelo cateter pré-lubrificado com bolsa, embora o resultado de ambos tenha sido satisfatório. $\mathrm{O}$ cateter convencional, fabricado com o mesmo material que o cateter pré-lubrificado com bolsa, obteve alta incidência de desconforto. Esse resultado leva a crer que sua ocorrência depende da sensibilidade individual, além da combinação entre lubrificação e flexibilidade do material do cateter. Em estudo realizado com crianças, 10\% dos participantes consideraram o cateter prélubrificado, hidrofílico, muito duro ${ }^{(9)}$. Já em estudo comparando diferentes cateteres hidrofílicos, o mesmo hidrofílico utilizado neste estudo obteve uma nota 9,6 da amostra para seu potencial de não causar dor, contra 8,7 dada para o cateter comparado.

Autores compararam dois cateteres hidrofílicos pré-lubrificados quanto ao desconforto, sendo um embalado com solução salina isotônica como o hidrofílico usado neste estudo e outro com necessidade de adicionar água antes da introdução, em 196 mulheres. Deste grupo, 98 mulheres referiram desconforto de diferentes tipos e intensidade, porém não houve diferença para os dois tipos de cateteres ${ }^{(14)}$.

Autores observaram uma preferência da amostra pelo cateter hidrofílico em relação ao não hidrofílico ${ }^{(10)}$. Vale considerar, novamente, que no estudo citado, o cateter hidrofílico também possuía reservatório de urina, o que pode ter contribuído para a diferença de resultados.

Para os indivíduos participantes de dois estudos, um internacional e um nacional ${ }^{(5-6)}$, o tempo necessário para a cateterização foi similar para cateter convencional e hidrofílico, assim como no presente estudo, não houve diferença estatisticamente significativa para o tempo de procedimento. Em outro estudo, o tempo de preparo para CIL com o cateter convencional e o hidrofílico foi o mesmo, com diferença para o tempo de introdução, que foi discretamente menor para o hidrofílico ${ }^{(9)}$.

Alguns autores observaram superioridade de resultado pelo cateter de lubrificação não hidrofílica quando comparado ao de lubrificação hidrofílica. Observando-se que os dois cateteres pré-lubrificados estudados não apresentavam bolsa coletora, pode-se sugerir que a superioridade de resultado pelo de lubrificação não hidrofílica, seja pelo fato de permitir um filme que o envolva e permita a técnica sem toque, dando mais firmeza à inserção ${ }^{(7)}$.

Observou-se neste estudo, assim como na maioria dos demais consultados, que os cateteres pré-lubrificados apresentam escores superiores, especialmente no que se refere à satisfação do usuário. Entretanto, o melhor material, a melhor lubrificação e atributos complementares dependem das necessidades, características e preferência do usuário.

\section{CONSIDERAÇÕES FINAIS}

Pode-se considerar como benefício do estudo a possibilidade dada aos participantes de conhecer e avaliar as tecnologias existentes para CIL. Espera-se que estes benefícios se estendam a outros usuários por meio da divulgação dos resultados e conscientização de profissionais e governantes quanto à necessidade e benefícios do direito de escolha do usuário.

Sugerem-se benefícios também às empresas fabricantes, tendo seus produtos avaliados pelo maior interessado, o usuário, possibilitando, a partir dos resultados, um aprimoramento de seus produtos de forma a responderem às reais necessidades de consumo.

Além do exposto, a pesquisa tem sua importância para os profissionais que atuam junto às pessoas com lesão medular em uso do CIL, no sentido de pensar as opções tecnológicas adequadas às condições daqueles que as utilizam e a orientação de uso apropriado da tecnologia, de forma a evitar complicações com a utilização e fornecer alternativas de acordo com as preferências do usuário.

Foi possível avaliar e discutir a satisfação do usuário com cada cateter e na comparação entre eles, respondendo às questões norteadoras. Porém, com a apresentação e discussão dos resultados, surgiram questões que podem direcionar pesquisas futuras, entre elas: o componente da pré-lubrificação, isoladamente, influencia na satisfação do usuário? O material com o qual o cateter foi produzido influencia na satisfação, desconsiderando outros aspectos? Itens complementares como anel de abertura da embalagem e picote para desprezar a urina da bolsa coletora são efetivamente utilizados 
pelo usuário? Destaca-se ainda a necessidade de pesquisas com maior tempo de seguimento, que permitam maior familiaridade do usuário com o cateter que está sendo avaliado.

Concluindo, a amostra estudada apresentou maiores níveis de satisfação com o conjunto para CILe com o cateter hidrofílico em comparação com o cateter convencional, porém, pela observação de que cada cateter agrada por aspectos diferentes, pode-se dizer que a indicação de um cateter ideal depende da avaliação individualizada do sujeito, suas dificuldades e potencialidades e suas preferências.

\section{AGRADECIMENTOS}

Agradecemos a toda a equipe das empresas Coloplast e BBraun por disponibilizarem os cateteres necessários para que esta pesquisa fosse possível e pela assessoria técnica dispensada antes, durante e após o período de estudo.

\section{REFERÊNCIAS}

1. Nardi AM, Costa E, Nardi AC, Nardozza A, Facio Júnior FN, de Faria GE et al: Hipogonadismo masculino tardio ou DAEM: diagnóstico. In: Nardi AC et al (ed.), Diretrizes urologia AMB. Rio de Janeiro, SBU Sociedade Brasileira de Urologia, 2014; 64-9.

2. Bezerra CA, Truzzi JC, Averbeck MA, Almeida SHM. Uroneurologia: Recomendações SBU 2013. SBU. 2013; 13-9.

3. Lapides J, Diokno AC, Silber SJ, Lowe BS. Clean Intermittent Self- catheterization in the treatment of urinary tract disease. J Uro. 1972; 167(2):458-461.

4. Vaidyanathan S, Soni BM, Singh G, Oo T, Hughes PL. Barriers to implementing intermittent catheterisation in spinal cord injury patients in Northwest Regional Spinal Injuries Centre, Southport, U.K. Scientific World Journal. 2011; (11):77-85.

5. de Ridder DJ, Evaraert K, Fernández LG, Valero $\mathrm{JV}$, Durán $\mathrm{AB}$, Abrisqueta $\mathrm{ML}$, et al. Intermittent catheterisation with hydrophilic-coated catheters (SpeediCath) reduces the risk of clinical urinary tract infection in spinal cord injured patients: a prospective randomised parallel comparative trial. Eur Urol. 2005; 48(6):991-5.

6. Martins MS, Santos VLCG, Secoli SR, Mata SM, Nogueira DS, Souza DM. Estudo comparativo sobre dois tipos de cateteres para cateterismo intermitente limpo em crianças estomizadas. Rev. esc. enferm. USP. 2009; 43(4):865-71. the use of conventional, hydrophilic and gel-lubricated catheters with regard to urethral micro trauma, urinary system infection, and patient satisfaction in patients with spinal cord injury: a randomized controlled study. Eur J Phys Rehab Med 2010; 46(4):473-9.

8. Almeida SO, Nique WM. Encantamento do cliente: proposição de uma escala para avaliação do constructo. Rev. adm. contemp. 2007; 11(4):109-30.

9. Boucher A, Cloutier J, Lebel S, Hamel M, Lamontagne $\mathrm{P}$, Bolduc S. Hydrophilic-coated catheter appreaciation study in a pediatric population. Can Urol Assoc J. 2010; 4(6):150-4.

10. Leriche A, Charvier K, Bonniaud V, Peyrat L, N'Guyen $\mathrm{P}$, Soler JM, et al. Etude d'acceptabilité du set de sondage SpeediCath ${ }^{\circledR}$ Set comparativement à Actreen ${ }^{\circledR}$ Set chez des patients pratiquant I'autosondage. Progrès en Urologie. 2006; 16:347-51.

11. Taskinen S, Fagerholm R, Ruutu M. Patient experience with hydrophilic catheters used in clean intermittent catheterization. J Pediatr Urol. 2008; 4(5):367-71.

12. Newman DK, Willson MM. Review of intermittent catheterization and current best practices. Urol Nurs 2011; 31(1):12-28.

13. Wyndaele J, de Ridder D, Everaert K, Heilporn A, Congard-Chassol B. Evaluation of the use of UrocathGel catheters for intermittent self-catheterization by male patients using conventional catheters for a long time. Spinal Cord. 2000; 38(2):97-9.

14. Litherland AT, Schiotz HA. Patient-perceived discomfort with two coated urinary atheters. Br J Nurs 2007; 16(5):284-7. 